STRUCTURAL BIOLOGY COMMUNICATIONS

ISSN 2053-230X
Received 23 November 2017

Accepted 4 January 2018

Edited by M. J. van Raaij, Centro Nacional de Biotecnología - CSIC, Spain

₹ Current address: School of Chemistry, University College Dublin, Stillorgan Road, Belfield, Dublin 4, Ireland.

Keywords: biofuels; glycosidases; enzymes; enzyme inhibitors; Talaromyces pinophilus; arabinofuranosidase.

PDB reference: Talaromyces pinophilus arabinofuranosidase, complex with AraDNJ, $6 f 1 j$

Supporting information: this article has supporting information at journals.iucr.org/f

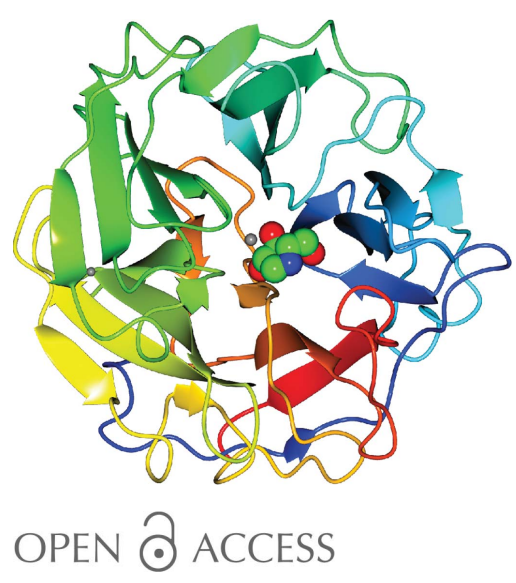

\section{Structure of a Talaromyces pinophilus GH62 arabinofuranosidase in complex with AraDNJ at $1.25 \AA$ resolution}

\author{
Olga V. Moroz, ${ }^{a}$ Lukasz F. Sobala, ${ }^{a}$ Elena Blagova, ${ }^{a}$ Travis Coyle, ${ }^{b} \ddagger$ Wei Peng, ${ }^{c}$ \\ Kristian B. R. Mørkeberg Krogh, ${ }^{d}$ Keith A. Stubbs, ${ }^{b}$ Keith S. Wilson a and \\ Gideon J. Davies ${ }^{\text {a* }}$
}

aYork Structural Biology Laboratory, Department of Chemistry, The University of York, York YO10 5DD, England,
${ }^{\mathbf{b}}$ School of Molecular Sciences, The University of Western Australia (M313), 35 Stirling Highway, Crawley, WA 6009,
Australia, 'Fungal Diversity, Novozymes A/S, China Headquarters, 14 Xinxi Road, Shangdi Zone, Haidian District,
Beijing 100085, People's Republic of China, and ${ }^{\mathbf{d} P r o t e i n ~ B i o c h e m i s t r y ~ a n d ~ S t a b i l i t y, ~ N o v o z y m e s ~ A / S, ~ K r o g s h ø j v e j ~ 36, ~}$
2880 Bagsværd, Denmark. *Correspondence e-mail: gideon.davies@york.ac.uk

The enzymatic hydrolysis of complex plant biomass is a major societal goal of the 21st century in order to deliver renewable energy from nonpetroleum and nonfood sources. One of the major problems in many industrial processes, including the production of second-generation biofuels from lignocellulose, is the presence of 'hemicelluloses' such as xylans which block access to the cellulosic biomass. Xylans, with a polymeric $\beta$-1,4-xylose backbone, are frequently decorated with acetyl, glucuronyl and arabinofuranosyl 'side-chain' substituents, all of which need to be removed for complete degradation of the xylan. As such, there is interest in side-chain-cleaving enzymes and their action on polymeric substrates. Here, the $1.25 \AA$ resolution structure of the Talaromyces pinophilus arabinofuranosidase in complex with the inhibitor AraDNJ, which binds with a $K_{\mathrm{d}}$ of $24 \pm 0.4 \mu M$, is reported. Positively charged iminosugars are generally considered to be potent inhibitors of retaining glycosidases by virtue of their ability to interact with both acid/base and nucleophilic carboxylates. Here, AraDNJ shows good inhibition of an inverting enzyme, allowing further insight into the structural basis for arabinoxylan recognition and degradation.

\section{Introduction}

The production of 'second-generation' biofuels, i.e. from nonfood plants, is a major societal goal as we move away from petroleum-based energy towards secure and renewable energy. Although the majority of polysaccharide biomass in plants is cellulose, the cellulose fibres are coated with hemicelluloses such as xylan, which render access to the cellulose more difficult. Enzymatic degradation of xylan is therefore necessary for the action of cellulase on higher plants, but it is also an important substrate in itself in that glucose and xylan, with small quantities of other sugars, are the major substrates for biofuel generation (discussed in Somerville, 2007). The enzymatic degradation of hemicelluloses such as xylan is of major importance in the biofuel industry (reviewed in Pauly \& Keegstra, 2008) and also in diverse industries such as bread manufacture, animal feed and the pulp and paper industry (for pulp bleaching). Xylan, which is a major component of the plant cell wall, consists of a backbone $\beta$-1,4-linked D-xylosyl chain, which is decorated with diverse substituents including 2and 3-linked arabinofuranosyl moieties (typically in cereal arabinoxylans) and glucuronic acid (notably in cereal and hardwood glucuronoxylans). Xylan complexity is further 
segmented through ester-linked species such as acetyl and ferulate species, with the latter potentially linking the xylan to lignin (Fig. 1a). Degradation of xylan both in natural environments and in the industrial milieu therefore requires a plethora of enzymes, with some of the main players including $\beta$-xylanases, $\beta$-xylosidases, $\alpha$-glucuronidases, acetyl and ferulate esterases and arabinofuranosidases, all of which are subject to keen academic and industrial study (recently comprehensively reviewed by Biely et al., 2016).

Arabinoxylans, by virtue of being found in many of the plants now favoured for biofuel production, are considered to be a major 'feedstock' if we are to attain these societal goals in terms of renewable and secure energy (for reviews, see, for example, Lagaert et al., 2014; Pauly \& Keegstra, 2008). Given that arabinoxylan degradation requires a consortium of enzymes acting in partial synergy, most elegantly emphasized through Gilbert's recent work on xylan degradation by the microbiota (Rogowski et al., 2015), there is much interest in the structure, mechanism and specificity of xylan-active enzymes, with a special focus on side-chain-cleaving enzymes and their potential synergy with backbone-cleaving xylanases. This potential synergy is further complicated by the differing capacities of the endoxylanases themselves to accommodate side chains. Of particular interest are the arabinofuranosidases, which are capable of removing the arabinofuranosyl (Araf) substituents from the 2- and 3-positions of the xylan backbone, thus opening up the xylan backbone for attack by classical endoxylanases. Arabinofuranosidases are found in families GH2, GH3, GH43, GH51, GH54 and GH62 of the CAZy sequence-based classification (http://www.cazy.org; Lombard et al., 2014).
CAZY family GH62 contains many enzymes that act as arabinoxylan-active arabinofuranosidases (extensively reviewed in Wilkens et al., 2017). The first three-dimensional structures of GH62 enzymes appeared in 2014, with structures reported from the bacteria Streptomyces coelicolor (Maehara et al., 2014) and S. thermoviolaceus (Wang et al., 2014) and of two fungal enzymes from Ustilago maydis and Podospora anserina (Siguier et al., 2014). The three-dimensional structures share a common five-bladed $\beta$-propeller fold with an active centre consistent with hydrolysis with inversion of anomeric configuration, with conserved Glu and Asp residues acting as the catalytic acid and catalytic base, respectively, in the single-displacement mechanism (Fig. 1b). GH62 enzymes have been reviewed in CAZYpedia (for a review, see The CAZypedia Consortium, 2018).

Here, we present the three-dimensional structure of a fungal GH62 arabinofuranosidase from Talaromyces pinophilus refined at $1.25 \AA$ resolution in complex with the bespoke iminosugar arabinofuranosidase inhibitor 1,4dideoxy-1,4-imino-L-arabinitol (AraDNJ). The complex sheds light on the active site and, in light of previously published data, allows analysis of how the enzyme interacts with arabinoxylan substrates, serving to remove these side chains from the xylan backbone.

\section{Materials and methods}

\subsection{Macromolecule production and small-molecule synthesis}

The enzyme (a single-module GH62 arabinofuranosidase with no predicted N-glycosylation sites; GenBank MG656406)

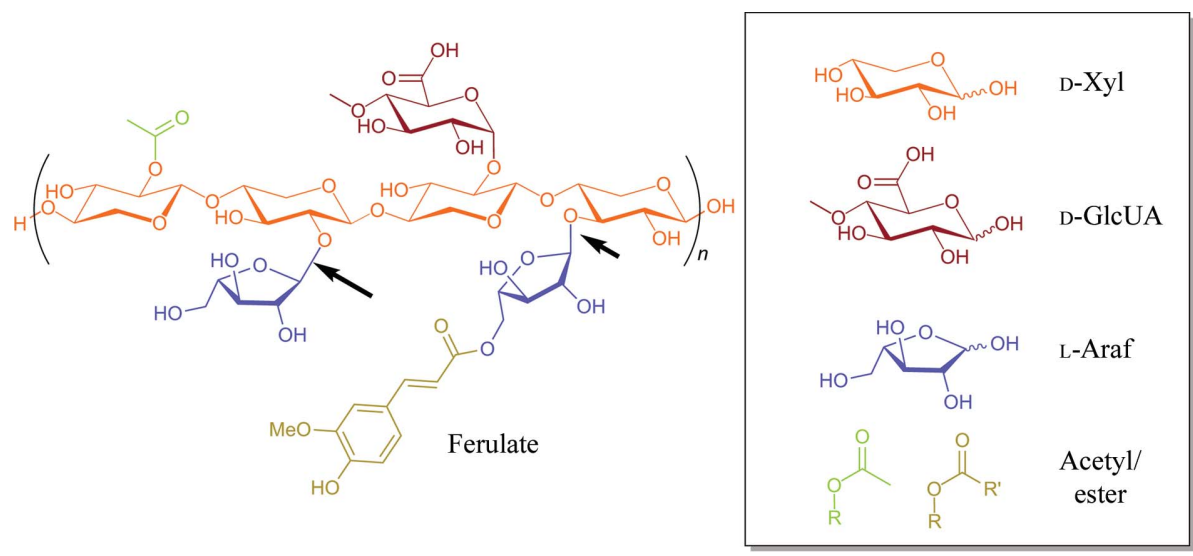

(a)
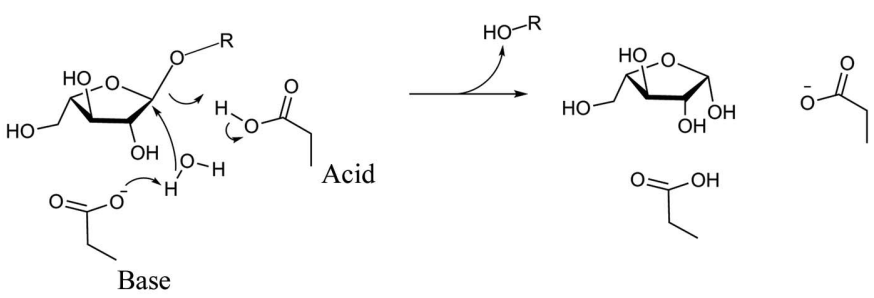

Figure 1

(b)

Xylans and their degradation. (a) The structure of a generic xylan, colour-coded by chemical group. Arrows indicate the positions of cleavage by arabinoxylan-active arabinofuranosidases. $(b)$ The reaction scheme for an inverting arabinofuranosidase, which requires the presence of both Brønsted acid and base residues. 
Table 1

Crystallization.

\begin{tabular}{ll}
\hline $\begin{array}{l}\text { Method } \\
\text { Plate type }\end{array}$ & $\begin{array}{l}\text { Vapour diffusion, sitting drop; MMS } \\
\text { MRC 2-well crystallization microplate, } \\
\text { Swissci, Switzerland }\end{array}$ \\
Temperature (K) & 293 \\
Protein concentration $\left(\mathrm{mg} \mathrm{ml}^{-1}\right)$ & 25 \\
Buffer composition of protein & $20 \mathrm{~m} M$ Tris-HCl pH $8.5,150 \mathrm{mM} \mathrm{NaCl}$ \\
$\quad$ solution & \\
Composition of reservoir solution & $30 \%$ PEG 2K MME, $0.2 \mathrm{M} \mathrm{KBr}$ \\
Volume and ratio of drop & $300 \mathrm{nl}$ total, $1: 1$ ratio \\
Volume of reservoir $(\mu \mathrm{l})$ & 54 \\
\hline
\end{tabular}

was cloned and expressed by standard heterologous expression at Novozymes A/S using Aspergillus oryzae as the expression host, essentially as discussed in Biely et al. (2014). A novel band of about $35 \mathrm{kDa}$ was observed in cultures of transformants that was not observed in cultures of the untransformed production strain. The expression level was investigated using SDS-PAGE for several transformants that appeared to express the recombinant arabinofuranosidase. After expression of the transformant with the highest expression level in a 11 bioreactor, the culture broth was sterile-filtered to remove the mycelia. The filtrated broth was brought to $1.8 \mathrm{M}$ ammonium sulfate, and after filtration $(0.22 \mu \mathrm{m}$ PES filter; Nalge Nunc International, Nalgene labware catalogue No. 595-4520) the filtrate was loaded onto a Phenyl Sepharose 6 Fast Flow column (high sub; GE Healthcare, Piscataway, New Jersey, USA) equilibrated with $25 \mathrm{~m} M$ HEPES pH 7.0 with $1.8 M$ ammonium sulfate; the column was washed with three column volumes of $25 \mathrm{mM}$ HEPES $\mathrm{pH}$ 7.0, 1.0 $M$ ammonium sulfate and bound proteins were eluted with $25 \mathrm{~m} M$ HEPES $\mathrm{pH}$ 7.0. The fractions were pooled and applied onto a Sephadex G-25 column (GE Healthcare) equilibrated with $25 \mathrm{~m} M$ HEPES pH 7.5. The fractions were applied onto a SOURCE 15Q column (GE Healthcare) equilibrated with $25 \mathrm{~m} M$ HEPES $\mathrm{pH} 7.5$ and the bound proteins were eluted with a linear gradient from 0 to $1000 \mathrm{~m} M$ sodium chloride over ten column volumes. Fractions were analyzed by SDS-PAGE and those containing the arabinofuranosidase were combined.

The synthesis of AraDNJ was carried out using literature procedures (Jones et al., 1985; Naleway et al., 1988).

\subsection{Crystallization}

Crystallization screening was carried out by sitting-drop vapour diffusion with drops set up using a Mosquito Crystal liquid-handling robot (TTP Labtech, England) with $150 \mathrm{nl}$ protein solution plus $150 \mathrm{nl}$ reservoir solution in 96-well format plates (MRC 2-well crystallization microplates, Swissci, Switzerland) equilibrated against $54 \mu \mathrm{l}$ reservoir solution. Experiments were carried out at room temperature using several commercial screens.

Extensive screening was carried out with no promising hits. As a final resort, the sample was subjected to shallow-gradient ion exchange in Tris- $\mathrm{HCl} \mathrm{pH}$ 8.5. The resultant peak was asymmetric and the conditions of the run were adjusted to optimize the separation of different regions of the peak
Table 2

Data-collection statistics.

Values in parentheses are for the outer shell.

\begin{tabular}{|c|c|}
\hline Diffraction source & I04-1, DLS \\
\hline Wavelength $(\AA)$ & 0.93 \\
\hline Temperature (K) & 100 \\
\hline Detector & PILATUS 6M-F \\
\hline Crystal-to-detector distance (mm) & 254.2 \\
\hline Rotation range per image $\left(^{\circ}\right)$ & 0.1 \\
\hline Total rotation range $\left({ }^{\circ}\right)$ & 180 \\
\hline Exposure time per image (s) & 0.0375 \\
\hline Space group & $P 2_{1}$ \\
\hline$a, b, c(\AA)$ & $43.83,88.97,72.66$ \\
\hline$\alpha, \beta, \gamma\left({ }^{\circ}\right)$ & $90,95.22,90$ \\
\hline Mosaicity $\left(^{\circ}\right)$ & 0.11 \\
\hline Resolution range $(\AA)$ & $33.52-1.25(1.27-1.25)$ \\
\hline Total No. of reflections & 457639 (14559) \\
\hline No. of unique reflections & $149344(6813)$ \\
\hline Completeness (\%) & $98(91)$ \\
\hline $\mathrm{CC}_{1 / 2}{ }^{\dagger}$ & $0.998(0.79)$ \\
\hline Multiplicity & $3.1(2.1)$ \\
\hline$\langle I / \sigma(I)\rangle$ & $13.1(2.9)$ \\
\hline$R_{\text {merge }}$ & $0.044(0.28)$ \\
\hline$R_{\mathrm{ri.m}}$ * & $0.052(0.34)$ \\
\hline Overall $B$ factor from Wilson plot $\left(\AA^{2}\right)$ & 5.1 \\
\hline
\end{tabular}

$\dagger \mathrm{CC}_{1 / 2}$ values for $I_{\text {mean }}$ are calculated by splitting the data randomly into two half data sets. $\$$ Estimated $R_{\text {r.i.m. }}=R_{\text {merge }}[N /(N-1)]^{1 / 2}$, where $N$ is the data multiplicity, and $R_{\text {merge }}$ is defined as $\sum_{h k l} \sum_{i}\left|I_{i}(h k l)-\langle I(h k l)\rangle\right| / \sum_{h k l} \sum_{i} I_{i}(h k l)$, where $I(h k l)$ is the intensity of the reflection.

(whole gradient $0-1 M \mathrm{NaCl}$, peak separation at $10-20 \%$ of elution buffer). Fractions for these regions were pooled separately and concentrated. Crystallization was set up with protein fractions from the beginning of the peak. Crystallizations were performed both with and without the inhibitor AraDNJ which, when used, was mixed with the protein to give a final concentration of $5 \mathrm{~m} M$. The best hit was obtained for protein in complex with the inhibitor from Crystal Screen HT condition G3 (0.01 $M$ zinc sulfate, $0.1 M$ MES pH 6.5, 25\% PEG $550 \mathrm{MME}$ ); this was chosen to make a seeding stock for further optimizations.

The seeding stock was prepared and microseed matrix screening (MMS; for a recent review, see D'Arcy et al., 2014) was carried out using an Oryx robot (Douglas Instruments) according to the published protocols (Shaw Stewart et al., 2011; Shah et al., 2005) with two screens, Crystal Screen HT and JCSG, as well as a number of optimizations of the hit conditions. Diffraction-quality crystals were obtained from JCSG screen conditions B2, G7 and G10. That used for data collection was obtained from condition G10, i.e. $30 \%$ PEG $2 \mathrm{~K}$ MME, $0.2 M \mathrm{KBr}$. The crystals were cryoprotected by adding PEG 3350 to the mother liquor in a 1:2 ratio $(3 \mu \mathrm{l} P E G+6 \mu \mathrm{l}$ mother liquor), which corresponded to $16.6 \%$ PEG 3350 and $20 \%$ PEG $2 \mathrm{~K}$ in the final cryoprotectant solution. Crystallization conditions are shown in Table 1.

\subsection{Data collection and processing}

All computations were carried out using programs from the CCP4 suite (Winn et al., 2011) unless otherwise stated. The data were collected on beamline I04-1 at Diamond Light Source (DLS) to $1.2 \AA$ resolution and were processed with 
xia2 (Winter et al., 2013). Data-collection and processing statistics are given in Table 2.

\subsection{Structure solution and refinement}

The structure was solved by MOLREP (Vagin \& Teplyakov, 2010) using $S$. coelicolor $\alpha$-L-arabinofuranosidase (PDB entry 3wmy; Maehara et al., 2014) as the search model. Chain tracing used Buccaneer, and the structure was refined with REFMAC (Murshudov et al., 2011) iterated with manual model correction using Coot (Emsley et al., 2010). The quality of the final model was validated using MolProbity (Chen et al., 2010) as part of the PHENIX package (Adams et al., 2011). The final refinement statistics are given in Table 3 . The structure has been deposited in the PDB as entry $6 \mathrm{f} 1 \mathrm{j}$.

\subsection{Isothermal titration calorimetry}

Ligand affinity was measured using isothermal titration calorimetry (ITC). ITC was performed at $25^{\circ} \mathrm{C}$ in $25 \mathrm{mM}$ HEPES pH 7.0, $100 \mathrm{~m} M \mathrm{NaCl}$ using a Malvern MacroCal Auto-iTC200 calorimeter. The ligand in the syringe was at $1.8 \mathrm{~m} M$ and was titrated into a cell containing a $112 \mu \mathrm{M}$ solution of the enzyme. Assays were performed in duplicate. The dissociation constant was calculated using the PEAQ-ITC Analysis software (Malvern).

\section{Results and discussion}

The structure (PDB entry $6 \mathrm{f} 1 \mathrm{j}$ ) was solved and refined at $1.25 \AA$ resolution (Table 3 ). The protein chain can be traced from residues 25 through to 325 and contains both structural calcium and zinc ions. The five-bladed $\beta$-propeller structure (Fig. 2a) bears a strong similarity to those of previously published GH62 enzymes, notably those from S. coelicolor (Maehara et al., 2014) and S. thermoviolaceus (Wang et al., 2014); 300 residues align with 72 and $69 \%$ sequence identity and r.m.s. $\mathrm{C}^{\alpha}$ deviations of 0.58 and $0.68 \AA$, respectively, as reflected by high PDBeFold (Krissinel \& Henrick, 2004) $Q$ scores of 0.95 and 0.94 , respectively. There are two subunits in the asymmetric unit with high structural similarity (r.m.s.d. of $0.22 \AA$ ), with some conformational differences on the outer surfaces, in particular in the region of crystal contacts.

Of the two metal ions, the $\mathrm{Ca}^{2+}$ ion is located essentially as reported previously, for example in the $S$. coelicolor enzyme (Maehara et al., 2014). However, this structural $\mathrm{Ca}^{2+}$ ion (which is close to, but does not impinge on, the active centre) is coordinated by six water molecules and a carboxylate $\mathrm{O}$ atom from Glu215. This is different to previous structures, in which the $\mathrm{Ca}^{2+}$ ion was coordinated by a His and Gln pair, which are replaced here by a water molecule hydrogenbonded to Ser278 (in place of the His) and directly to Glu215 (in place of the Gln observed previously). In the T. pinophilus enzyme there are additional $\mathrm{Zn}^{2+}$ ions derived from the 'seeding stock' (see above) element of the crystallization conditions. One of those bridges the $A$ and $B$ molecules in the lattice, presumably aiding lattice formation, with coordination from His180 from molecule $A$ and the amino-terminal $\mathrm{NH}_{2}$
Table 3

Structure solution and refinement.

\begin{tabular}{ll}
\hline Resolution range $(\AA)$ & $33.52-1.25$ \\
Completeness $(\%)$ & 97.8 \\
No. of reflections & \\
Working set & 141792 \\
Test set & 7088 \\
Final $R_{\text {cryst }}$ & 0.120 \\
Final $R_{\text {free }}$ & 0.136 \\
Cruickshank DPI & 0.037 \\
No. of subunits in the asymmetric unit & 2 \\
R.m.s. ${ }^{\alpha}$ deviation between subunits $(\AA)$ & 0.221 \\
No. of non-H atoms & \\
Protein & 4698 \\
Ion & 4 \\
Ligand & 18 \\
Water & 658 \\
Total & 5378 \\
R.m.s. deviations & \\
Bonds $(\AA)$ & $0.014(0.020)$ \\
Angles $\left({ }^{\circ}\right)$ & $1.5(1.9)$ \\
Average $B$ factors $\left(\AA^{2}\right)$ & \\
Protein & \\
$\quad$ Chain $A$ & 7.3 \\
Chain $B$ & 7.7 \\
Ions & \\
$\quad$ Ca & \\
Zn & \\
Zn ${ }^{2+}(1$ st $)$ & 3.3 \\
Ligand & 8.8 \\
Water & 8.4 \\
Ramachandran plot $\dagger$ & 6.7 \\
Favoured $(\%)$ & 18.8 \\
Outliers $(\%)$ & 96.4 \\
MolProbity score & 0.33 \\
\hline$\quad$ & 0.85 \\
\hline
\end{tabular}

$\dagger$ Ramachandran plot analysis was carried out by MolProbity (Chen et al., 2010).

and carbonyl groups of Ser24 and the side chain of Glu220 from molecule $B$. Another $\mathrm{Zn}^{2+}$ ion is coordinated by Glu 88 from molecule $B$, His 180 from the symmetry-related molecule $B$ and three waters.

The structure of the $T$. pinophilus GH62 arabinofuranosidase was determined in the presence of the putative arabinofuranosidase inhibitor AraDNJ (Fig. 2b), which allows further confirmation of the catalytic apparatus. This compound has found use in studies of other arabinofuranosidases (Axamawaty et al., 1990; Hemsworth et al., 2016) as well as as a scaffold for developing inhibitors of other glycosidases (Siguier et al., 2014; Mena-Barragán et al., 2016). Azasugars and iminosugars are generally considered to be good inhibitors of retaining glycoside hydrolases by virtue of their endocyclic $\mathrm{N}$ atom, which can be protonated, thus mimicking the putative positive charge that is thought to exist in the transition state(s) during glycoside hydrolysis. In addition, the $\mathrm{N}$ atom provides adventitious interactions with both the acid/base and the nucleophile in the active sites of these enzymes (see, for example, Gloster et al., 2007). GH62 enzymes are inverting and thus do not have a suitably positioned nucleophile. It was therefore surprising to us that AraDNJ acted as an inhibitor with well resolved density. The binding constant for AraDNJ was therefore determined by isothermal titration calorimetry (Fig. $2 c$ ), revealing a surprisingly tight $K_{\mathrm{d}}$ of $24 \pm 0.4 \mu \mathrm{M}$. It is rare in glycosidases that iminosugars bind so well to the glycosidase active site without 
a close enzyme-derived nucleophilic interaction, but other examples include CAZY family GH6, where cellobio-derived isofagomines have been used to good effect, even reporting on the substrate distortions involved in catalysis (Gloster et al., 2007). Here, AraDNJ binds in a potentially transition-statemimicking ${ }^{4} E$ conformation. As might be expected, AraDNJ binds in the same location as observed for Araf itself (see, for example, PDB entry 4o8o; Wang et al., 2014), making similar hydrogen bonds from $\mathrm{O} 2$ and $\mathrm{O} 3$ to Asp160, from $\mathrm{O} 3$ to Gln120 and from O56 to Asp52. There is also a potential hydrophobic contact with the side chain of Ile159. There is no direct interaction of the positively charged $\mathrm{N}$ atom (here replacing the endocyclic $\mathrm{O}$ atom of arabinose), but the structure reveals a water molecule poised $3.1 \AA$ 'below' the furanose ring, where it hydrogen-bonds to Asp52, the putative catalytic base, consistent with previous studies (Maehara et al., 2014; Wang et al., 2014) and the inverting mechanism (Fig. 1b). Glu212, the putative acid, is placed for lateral anti protonation of any departing group (Fig. 2d). Notably, the positively charged $\mathrm{N}$ atom lies exactly where the positively charged $\mathrm{N}$ atom of published Tris complexes of homologues sits (see, for example, PDB entry $3 \mathrm{wn} 2$, the S. coelicolor GH62 enzyme; Maehara et al., 2014), highlighting that these enzymes have evolved to stabilize the positively charged transition state, even without the aid of the direct charge-charge interactions available to retaining enzymes.

The T. pinophilus GH62 enzyme in complex with AraDNJ, viewed in light of past work on xylooligosaccharide complexes of GH62 enzymes, provides further insight into the mechanisms by which GH62 enzymes remove the arabinofuranoside

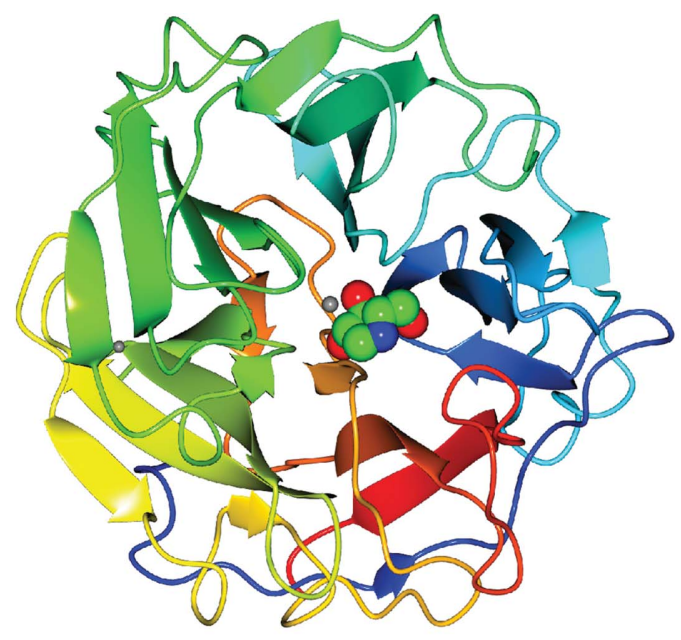

(a)

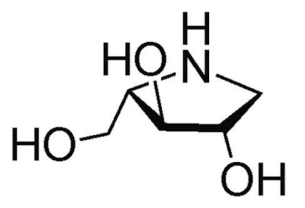

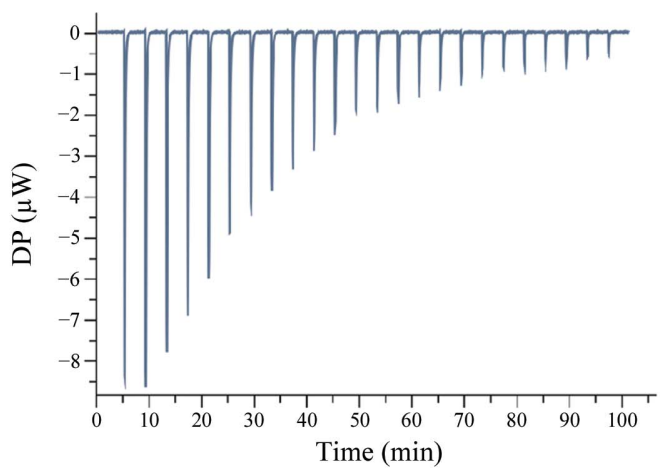

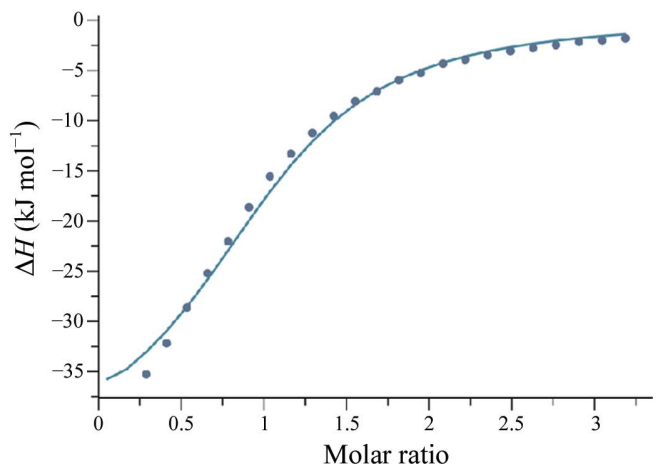

(c)

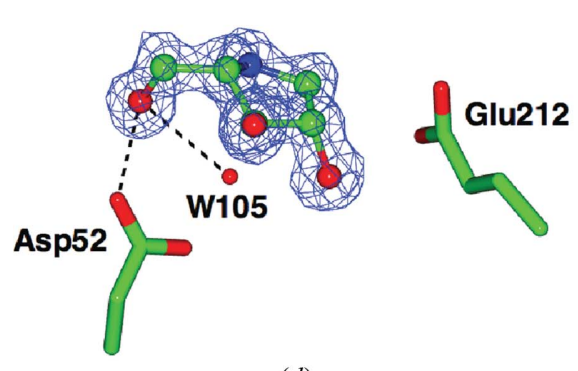

(d)
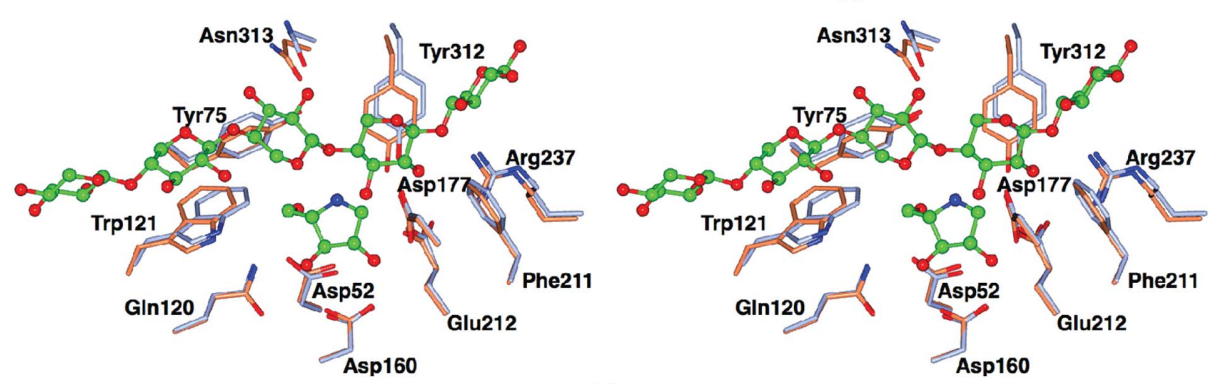

(e)

Figure 2

Three-dimensional structure and ligand binding of the T. pinophilus GH62 arabinofuranosidase in complex with the inhibitor AraDNJ. (a) Threedimensional structure colour-ramped from the N-terminus (blue) to the C-terminus (red). Metal ions are shown as shaded spheres and AraDNJ as a CPK model. (b) The chemical structure of AraDNJ. (c) ITC data for AraDNJ binding $\left(K_{\mathrm{d}}\right.$ of $\left.24 \pm 0.4 \mu M\right)$. (d) Observed electron density for AraDNJ bound to GH62, $2 F_{\mathrm{o}}-F_{\mathrm{c}}$ (maximum-likelihood/ $\sigma_{\mathrm{A}}$-weighted) at $1.25 \AA$ contoured at $1 \sigma$. The catalytic acid Glu212 and base Asp52 are shown, along with a water molecule poised for nucleophilic attack. (e) Partial overlay of the T. pinophilus GH62 arabinofuranosidase (brown with AraDNJ in green) with the S. coelicolor GH62 arabinofuranosidase (PDB entry $3 \mathrm{wn} 2$; pale blue with xylopentaose in green), highlighting the highly conserved binding centre and the recognition apparatus for the arabinoxylan chain. Structural figures were drawn with CCP4mg (McNicholas et al., 2011). 
decorations from arabinoxylan. An overlay with the xylopentaose complex (PDB entry 3wn2) of the $S$. coelicolor GH62 enzyme (Maehara et al., 2014; Fig. 2e) shows how the interacting surface for the xylan chain is highly conserved between the two enzymes, with both aromatic platforms (Phe211, Tyr312 and Trp121) and some hydrogen-bonding interactions (Arg237, Asn313 and Asp177) being invariant, suggesting that ligand recognition is similar. Indeed, $\mathrm{C} 1$ of the AraDNJ complex lies $1.9 \AA$ from the $\mathrm{O} 3$ atom of the 'second' (from the reducing end) xylose moiety in PDB entry $3 \mathrm{wn} 2$, highlighting how the T. pinophilus GH62 enzyme could act as an arabinofuranosidase that is active on O3-substituted xylans, as was proposed originally for the $S$. coelicolor GH62 enzyme (Maehara et al., 2014), although it is possible to also consider action at the $\mathrm{O} 2$ position should the xylan chain occasionally be reversed through the active site (which is possible with xylans given their internal pseudo-symmetry).

The T. pinophilus GH62 enzyme thus adds to the growing literature surrounding these key players in natural and industrial arabinoxylan degradation. It demonstrates how arabinofuranoside mimics lie in the active site of the enzyme and how the enzyme recognizes and cleaves arabinoxylan. Furthermore, the nonclassical application of an iminosugarbased glycosidase inhibitor to study inverting-enzyme structure and function should encourage the further non-intuitive application of such compounds in the future.

\section{Acknowledgements}

The authors would like to thank Novozymes A/S for partially funding this work. GJD is a Royal Society Ken Murray Research Fellow. KAS thanks the Australian Research Council and TC thanks the Australian Government, the University of Western Australia (UWA) and the Centre for Microscopy, Characterization and Analysis at UWA. The authors would also like to thank Diamond Light Source for beamtime (proposal mx13587) and the staff of beamline I04 for assistance with crystal testing and data collection.

\section{References}

Adams, P. D. et al. (2011). Methods, 55, 94-106.

Axamawaty, M. T., Fleet, G. W., Hannah, K. A., Namgoong, S. K. \& Sinnott, M. L. (1990). Biochem. J. 266, 245-249.

Biely, P., Puchart, V., Stringer, M. A. \& Mørkeberg Krogh, K. B. R. (2014). FEBS J. 281, 3894-3903.
Biely, P., Singh, S. \& Puchart, V. (2016). Biotechnol. Adv. 34, 12601274.

Chen, V. B., Arendall, W. B., Headd, J. J., Keedy, D. A., Immormino, R. M., Kapral, G. J., Murray, L. W., Richardson, J. S. \& Richardson, D. C. (2010). Acta Cryst. D66, 12-21.

D’Arcy, A., Bergfors, T., Cowan-Jacob, S. W. \& Marsh, M. (2014). Acta Cryst. F70, 1117-1126.

Emsley, P., Lohkamp, B., Scott, W. G. \& Cowtan, K. (2010). Acta Cryst. D66, 486-501.

Gloster, T. M., Meloncelli, P., Stick, R., Zechel, D., Vasella, A. \& Davies, G. J. (2007). J. Am. Chem. Soc. 129, 2345-2354.

Jones, D. W. C., Nash, R. J., Bell, E. A. \& Williams, J. M. (1985). Tetrahedron Lett. 26, 3125-3126.

Hemsworth, G. R., Thompson, A. J., Stepper, J., Sobala, Ł. F., Coyle, T., Larsbrink, J., Spadiut, O., Goddard-Borger, E. D., Stubbs, K. A., Brumer, H. \& Davies, G. J. (2016). Open Biol. 6, 160142.

Krissinel, E. \& Henrick, K. (2004). Acta Cryst. D60, 2256-2268.

Lagaert, S., Pollet, A., Courtin, C. M. \& Volckaert, G. (2014). Biotechnol. Adv. 32, 316-332.

Lombard, V., Golaconda Ramulu, H., Drula, E., Coutinho, P. M. \& Henrissat, B. (2014). Nucleic Acids Res. 42, D490-D495.

Maehara, T., Fujimoto, Z., Ichinose, H., Michikawa, M., Harazono, K. \& Kaneko, S. (2014). J. Biol. Chem. 289, 7962-7972.

McNicholas, S., Potterton, E., Wilson, K. S. \& Noble, M. E. M. (2011). Acta Cryst. D67, 386-394.

Mena-Barragán, T., García-Moreno, M. I., Nanba, E., Higaki, K., Concia, A. L., Clapés, P., García Fernández, J. M. \& Ortiz Mellet, C. (2016). Eur. J. Med. Chem. 121, 880-891.

Murshudov, G. N., Skubák, P., Lebedev, A. A., Pannu, N. S., Steiner, R. A., Nicholls, R. A., Winn, M. D., Long, F. \& Vagin, A. A. (2011). Acta Cryst. D67, 355-367.

Naleway, J. J., Raetz, C. R. \& Anderson, L. (1988). Carbohydr. Res. 179, 199-209.

Pauly, M. \& Keegstra, K. (2008). Plant J. 54, 559-568.

Rogowski, A. et al. (2015). Nature Commun. 6, 7481.

Shah, A. K., Liu, Z.-J., Stewart, P. D., Schubot, F. D., Rose, J. P., Newton, M. G. \& Wang, B.-C. (2005). Acta Cryst. D61, 123-129.

Shaw Stewart, P. D., Kolek, S. A., Briggs, A. R., Chayen, N. E. \& Baldock, P. F. M. (2011). Cryst. Growth Des. 11, 3432-3441.

Siguier, B., Haon, M., Nahoum, V., Marcellin, M., Burlet-Schiltz, O., Coutinho, P. M., Henrissat, B., Mourey, L., O'Donohue, M. J., Berrin, J.-G., Tranier, S. \& Dumon, C. (2014). J. Biol. Chem. 289, 5261-5273.

Somerville, C. (2007). Curr. Biol. 17, R115-R119.

The CAZypedia Consortium (2018). Glycobiology, 28, 3-8.

Vagin, A. \& Teplyakov, A. (2010). Acta Cryst. D66, 22-25.

Wang, W., Mai-Gisondi, G., Stogios, P. J., Kaur, A., Xu, X., Cui, H., Turunen, O., Savchenko, A. \& Master, E. R. (2014). Appl. Environ. Microbiol. 80, 5317-5329.

Wilkens, C., Andersen, S., Dumon, C., Berrin, J.-G. \& Svensson, B. (2017). Biotechnol. Adv. 35, 792-804.

Winn, M. D. et al. (2011). Acta Cryst. D67, 235-242.

Winter, G., Lobley, C. M. C. \& Prince, S. M. (2013). Acta Cryst. D69, 1260-1273. 